\title{
Autoestima y consumo de tabaco en adolescentes de secundaria
}

Laura Hinojosa-García (D) 1; Pedro García-García (DD 2*; Aída Aleyda Jiménez-Martínez (D) 3;

Gloria Nelly Gracia-Castillo (D) 4

\section{RESUMEN}

Introducción: El abuso de sustancias psicoactivas, es hoy en día un problema de salud pública muy vinculado a la etapa de la adolescencia por no estar aun completamente definida la personalidad y autoestima. Objetivos: Los objetivos del estudio fueron determinar la prevalencia de consumo de tabaco en adolescentes, identificar niveles de autoestima y la relación del nivel de autoestima con el consumo de tabaco. Metodología: Estudio descriptivo, correlacional y transversal, en una población de 900 adolescentes, muestreo no probabilístico por conveniencia para una muestra final de 324 adolescentes de ambos sexos. Fueron utilizadas, la Escala de Autoestima de Rosenberg y las prevalencias de consumo de tabaco global, lápsica, actual e instantánea. Resultados: La media de edad de los participantes fue de 13.3 años $(D E=0.99)$, el 54 \% de los participantes correspondió al género femenino y el $46 \%$ al masculino. La prevalencia de consumo de tabaco alguna vez en la vida fue del $9.6 \%$, mientras que en el último mes fue de $1.9 \%$. El $62.3 \%$ de los adolescentes se ubicaron en el rango de autoestima alta; el porcentaje encontrado en los masculinos fue mayor (70.5 \%), en comparación con el femenino (55.4 \%). No se documentó relación significativa entre los niveles de autoestima y el consumo de tabaco $(p>$.05). Conclusiones: La prevalencia y la frecuencia en el consumo de tabaco reportada fue muy baja. Se identificó que el mayor porcentaje de los participantes en el estudio, mostraron niveles de autoestima alto.

Palabras clave: Autoestima; Consumo de tabaco; Adolescentes (DeCS).

${ }^{1}$ Doctora en Ciencias de Enfermería. Universidad Autónoma Tamaulipas, Unidad Académica Multidisciplinaria Matamoros.

${ }_{2}^{2}$ Maestro en Ciencias de Enfermería. Universidad Autónoma Tamaulipas, Unidad Académica Multidisciplinaria Matamoros.

${ }^{3}$ Doctora en Ciencias de Enfermería. Universidad Autónoma de Tamaulipas, Unidad Académica Multidisciplinaria Matamoros.

${ }^{4}$ Maestra en Comunicación Académica. Universidad Autónoma Tamaulipas Unidad Académica Multidisciplinaria Matamoros.

Recibido: $30 / 01 / 2020$

Aceptado: $24 / 06 / 2020$

* Autor para correspondencia: pedro-2122@hotmail.com

Copyright 02020 SANUS

Artículo de acceso abierto distribuido

bajo Licencia Creative Commons

\section{cc) (i) $\Theta$

\section{Cómo citar este artículo}

Hinojosa-García L, García-García P, Jiménez-Martínez AA, Gracia-Castillo GN. Autoestima y consumo de tabaco en adolescentes de secundaria. SANUS. 2020; (14): 1-10. [Acceso____ ]; Disponible en: 


\title{
Self-esteem and tobacco consumption in high school adolescents
}

\begin{abstract}
Introduction: The abuse of psychoactive substances is nowadays a public health problem closely linked to the adolescence stage due to the fact that both personality and self-esteem are not yet fully defined. Objectives: The objectives of this study are to determine the prevalence of tobacco consumption in adolescents, identify levels of self-esteem, and the relationship of the level of self-esteem with tobacco consumption. Methodology: This was a cross-sectional-descriptive and correlational study applied on a population of 900 adolescents with non-probability convenience sampling for a final sample of 324 adolescents of both genders. The Rosenberg Self-Esteem Scale and the prevalence of global, lapsic, current, and instantaneous tobacco consumption were used. Results: The average age of the participants was 13.3 years (SD =0.99); $54 \%$ of the participants were females and $46 \%$ were males. The prevalence of tobacco consumption at some time in their life was $9.6 \%$, while in the last month it was 1.9\%. 62.3\% of the adolescents were located in the high self-esteem range; with a higher percentage in male participants (70.5\%), compared to female participants (55.4\%). There was no significant relationship between levels of self-esteem and smoking ( $p>$.05). Conclusions: The prevalence and frequency of tobacco consumption reported was very low. It was found that the highest percentage of participants in the study had high self-esteem.
\end{abstract}

Key words: Self-esteem; Tobacco consumption; Adolescents (DeCS). 


\title{
Auto-estima e consumo de tabaco em adolescentes secun- dária
}

\begin{abstract}
ABSTRATO
Introdução: Atualmente, o abuso de substâncias psicoativas é um problema de saúde pública intimamente ligada ao estágio da adolescência, porque a personalidade e a auto-estima ainda não estão completamente definidas. Objetivo: os objetivos do estudo foram determinar a prevalência do consumo de tabaco em adolescentes, identificar os níveis de auto-estima e a relação do nível de auto-estima com o consumo de tabaco. Metodologia: Estúdio descritivo, correlacional e transversal, aplicado em uma população de 900 adolescentes com amostragem não probabilística por conveniência, para uma amostra final de 324 adolescentes de ambos os sexos. Utilizou-se a Escala de Auto-Estima de Rosenberg e a prevalência de consume global, lapícico, atual e instantâneo de tabaco. Resultados: A média de idade dos participantes foi de 13.3 anos ( $\mathrm{DP}=$ = 9,99), 54\% dos participantes corresponderam ao sexo feminino e 46\% ao masculino. A prevalência do consumo de tabaco em algum momento da vida foi de $9.6 \%$, enquanto no último mês foi de 1,9\%. 62,3\% dos adolescentes estavam na faixa de auto-estima alta; o percentual encontrado no sexo masculino foi maior (70,5\%), em comparação às participantes do sexo feminino (55,4\%). Não houve relação significativa entre os níveis de auto-estima e consumo de tabaco. $(p>0,05)$. Conclusões: A prevalência de frequência de consumo de tabaco relatadas foram muito baixas. A prevalência de frequência de consumo de tabaco relatadas foram muito baixas. Verificuou-se que o maior percentual da participantes do estudo apresentava alta auto-estima.
\end{abstract}

Palavras chave: Simulação, estudantes de enfermagem, laboratório, enfermagem (DeCS). 


\section{INTRODUCCIÓN}

El consumo de tabaco hoy en día es considerado un grave problema social y de salud pública, se calcula que casi 6 millones de personas mueren al año en el mundo por enfermedades atribuidas al consumo de tabaco (1). Según datos de la Organización Mundial de la Salud(2) (2018), el tabaco es una de las principales amenazas para la salud pública en el mundo, más de 7 millones de personas al año mueren a causa de su consumo, de las cuales más de 6 millones son consumidores activos y alrededor de 890000 son no fumadores, expuestos al humo ajeno. El consumo de tabaco está relacionado con el riesgo de mortalidad por enfermedades como el cáncer, cardiopatía isquémica, enfermedad pulmonar obstructiva crónica y accidente cerebrovascular entre las más comunes ${ }^{(3)}$.

De acuerdo con la Encuesta Mundial sobre Tabaquismo en Jóvenes publicada en el 2016(4), a nivel global, aproximadamente 9.5 millones de jóvenes entre 13 a 15 años consumen tabaco, mientras que en el continente americano la prevalencia de consumo de tabaco en adolescentes de entre 13 a 15 años es de $15 \%$. Los países suramericanos presentan datos más elevados en el consumo de cigarri\|los, en tanto que en el área de Norteamérica se observó una disminución considerable en el consumo de cigarrillos del 36.4 \% encontrado en 1997, a $23 \%$ para el 2007(5). En lo que respecta a México, de acuerdo con la Encuesta Nacional de Consumo de Drogas, Alcohol y Tabaco 2016$2017^{(6)}, 14.9$ millones de mexicanos son fumadores actuales (3.8 millones son mujeres y 11.1 millones son hombres) de los cuales, 5.4 millones fuman diariamente. Mientras que, la prevalencia global de consumo de tabaco en adolescentes mexicanos es de $4.9 \%$ y la edad promedio de inicio de consumo de tabaco diario es de 14.3 años.

Existen antecedentes de que la mayoría de los adultos fumadores inician con el consumo durante la etapa de la adolescencia, como consecuencia de diversos factores como el emocional, el cual, se establece como una causa y un efecto cada vez más claro en la literatura. Diversos estudios evidencian direccionalidad entre consumo de tabaco y malestares emocionales tales como preocupación, miedo y estado de ánimo decaído en la adolescencia, aunque aún no se tiene claro sobre la relación causal de esta asociación ${ }^{(7)}$. No obstante, existe evidencia de que los adolescentes con buenas competencias emocionales presentan una mayor autoestima y un ajuste psicológico adecuado en cuanto a sus emociones y comportamiento ${ }^{(8)}$.

La adolescencia es la población más vulnerable para el inicio del consumo de tabaco ya que, aproximadamente el 90\% de los fumadores adultos han iniciado el hábito durante esta etapa ${ }^{(9)}$. Asimismo, las características típicas de la edad, la curiosidad de contar con nuevas experiencias de vida, así como dificultades dentro del ámbito familiar y escolar, son factores que pueden desencadenar que el adolescente pruebe el primer cigarrillo, el primer trago de alcohol e incluso la primera droga ilegal. En los países occidentales, más de la mitad de los adolescentes han tenido la experiencia de consumir tabaco durante su educación secundaria ${ }^{(10)}$. Algunos estudios sobre drogas muestran que el uso casual o permanente de alcohol y tabaco, solos o combinados es común entre los adolescentes, con prevalencias mayormente en los hombres ${ }^{\left({ }^{11}\right)}$ y con un número más alto de usuarios de alcohol que de tabaco ${ }^{\text {(12). }}$.

La adolescencia es considerada como una etapa especialmente relevante para la formación de la autoestima, debido a los cambios biológicos, psicológicos, sociales y cognitivos que se presentan durante este ciclo. La adolescencia constituye un periodo importante para el desarrollo del individuo, y es una etapa en la cual se afianza y consolida su personalidad; razón por la cual, es importante la transmisión de hábitos saludables que actúen como factores protectores en la transición hacia la adultez ${ }^{(13)}$.

Rosenberg (14), define la autoestima como el conjunto de pensamientos y sentimientos de un individuo sobre su propio valor e importancia, esta definición refleja la noción de autoestima "global" o "general". La autoestima es una parte importante en la autocomprensión de los adolescentes, y es probable que sea una construcción dinámica y fluctuante, susceptible a las influencias internas y externas durante la adolescencia. Un nivel alto de autoestima es considerado un factor protector que aparta al sujeto de las influencias no saludables, por lo que contrariamente, la autoestima baja implica autorechazo, autoinsatisfacción, autodesprecio y de esta forma el individuo carece de estima hacia el mismo, lo que puede colocar al sujeto en vulnerabilidad de conductas riesgosas como sería el consumo de sustancias.

La literatura existente que aborda la relación de la autoestima y los estilos de vida saludable es aún confusa y contradictoria. Por una parte, diversos estudios respaldan la hipótesis de que, a mayor nivel de autoestima, menor es el riesgo de conductas no saludables como el consumo de sustancias $(15,16)$. Un estudio realizado con el objetivo de estudiar los factores psicosociales que se asocian al consumo de sustancias como tabaco, alcohol y marihuana en una muestra de estudiantes universitarios mexicanos y colombianos, identificaron a la autoestima positiva como un factor protector ante el consumo de sustancias ${ }^{(17)}$. En este sentido, estudios previos identifican que, ante un nivel bajo de autoestima, mayor es el riesgo de consumo de diversos tipos de sustancias psicoactivas ${ }^{(18,19)}$. Lo anterior, pudiera confirmar el hecho de que, tener una autoestima alta o media, es útil para evitar conductas que dañen la salud del adolescente, como es el uso y abuso del consumo de drogas tanto legales como ilegales. Sin embargo, en un estudio realizado en una muestra de adolescentes en Cartagena Colombia, no se demostró asociación significativa de una baja autoestima con el consumo de cigarrillo ${ }^{(5)}$.

Por lo anteriormente referido, se identifica la necesidad de profundizar en el estudio sobre el fenómeno descrito y se 
plantean los siguientes objetivos de estudio: determinar los niveles de autoestima en adolescentes de secundaria, identificar la prevalencia global, lápsica, actual e instantánea de consumo de tabaco en adolescentes de secundaria, comprobar la relación entre la autoestima y el consumo de tabaco.

\section{METODOLOGÍA}

El diseño del estudio fue de tipo descriptivo, transversal, correlacional y el muestreo no probabilístico por conveniencia. La población estuvo constituida por 900 estudiantes de nivel secundaria y la muestra estuvo representada por 324 adolescentes de ambos géneros, que cumplieran con el criterio de inclusión, es decir, estar matriculados en el ciclo escolar 20172018 y estar de acuerdo en participar en el estudio.

\section{Instrumentos}

Se aplicó una Cédula de Datos Personales y Consumo de Tabaco (CDPCT) y un instrumento, la CDPCT está compuesta por información personal del participante como edad, género, grado escolar, estado civil y ocupación. Además incluye 5 reactivos que miden la edad de inicio de consumo, así como la prevalencia de consumo de tabaco global, lápsica, actual e instantánea .

Para medir la autoestima se utilizó la Escala de Autoestima de Rosenberg (1965) ${ }^{(14)}$, la cual permite explorar la autoestima personal entendida como los sentimientos de valía personal y de respeto a sí mismo. Está integrado por diez reactivos frases de las que cinco están enunciadas de forma positiva que comprende los reactivos del 1 al 5; mientras que los reactivos que se puntúan de forma negativa son del 6 al 10.

La escala tiene como puntaje global máximo 40 puntos y mínimo 10 puntos, donde a mayor puntaje mayor autoestima. De acuerdo a la escala, el nivel de autoestima se clasifica en función de tres puntos de corte: nivel de autoestima elevada o normal si se obtiene de 30 a 40; autoestima media si se obtiene de 26 a 29 puntos, y es indicativo de que no se presentan problemas de autoestima grave, sin embargo se recomienda mejorarla, finalmente si el puntaje obtenido es menor de 25 es evaluado como un nivel de autoestima baja y se considera que existen problemas de autoestima significativos. Esta escala ha sido utilizada medir la autoestima en adolescentes mexicanos de nivel secundaria ${ }^{(20)}$ reportando una confiabilidad aceptable de .89; así como en una muestra de universitarios de CDMX (21) encontrando un Alpha de Cronbach de .79 .

\section{Procedimiento de recolección de datos}

Se contó con la aprobación del comité de Investigación y Ética de la Unidad Académica Multidisciplinaria Matamoros-UAT (número de registro 054), así como de la dirección de la institución educativa donde fueron colectados los datos. Se abordó a los alumnos en un espacio en el cual estuvieran cómodamente sentados, se les explicó el objetivo del estudio y el otorgamiento del asentimiento y consentimiento informado para adolescentes y padres de familia en caso de que decidieran ser parte del estudio, aclarándoles que tenían la libertad de retirarse del estudio en el momento que así lo consideraran necesario. Posteriormente, se les entregó un sobre conteniendo la CDPYCT y el instrumento para la medición de la variable de estudio, al finalizar se les agradeció su participación.

\section{Consideraciones éticas}

El estudio se apegó a lo dispuesto por la Ley General de Salud en Materia de Investigación en el área de la salud

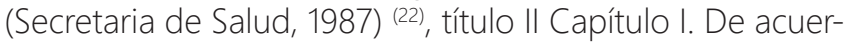
do con el artículo 13 se respetó la dignidad de los sujetos mediante un trato respetuoso y profesional y la protección de sus derechos humanos, conforme a lo establecido en el artículo 14 (Fracción V, VII y VIII) se ajustó a principios científicos y éticos, además se contó con el consentimiento informado por escrito del padre, madre o tutor además del participante, así como la garantía de recibir respuesta a cualquier pregunta respecto a los procedimientos, la seguridad de que no se identificará al sujeto y que se mantendrá la confidencialidad de la información relacionada con su privacidad, de igual forma que tendrá la oportunidad de retirar su consentimiento informado cuando así lo deseara (artículo 21, fracción I, II, IV, VI, VII y VIII).

\section{Análisis de resultados}

Los datos obtenidos de la recolección fueron procesados a través del programa estadístico SPSS versión 22.0. Primeramente, se revisó la consistencia interna del instrumento de Rosenberg a través del Coeficiente de Confiabilidad de Alpha de Cronbach. Posteriormente se utilizó estadística descriptiva e inferencial. El análisis descriptivo de las variables continuas y categóricas se realizó mediante frecuencias, proporciones y medidas de tendencia central y de variabilidad. Para determinar asociación se utilizó Chi-cuadrada de Pearson.

\section{RESULTADOS}

Para determinar la consistencia interna de la Escala de Autoestima de Rosenberg (1965), se calculó el Coeficiente Alpha de Cronbach, obteniendo una confiabilidad de .81. En relación a las variables sociodemográficas de los participantes del estudio, estos presentaron una media de edad de 13.3 años (DE = 0.99) con una mínima de 12 años y una máxima de 15 años. En cuanto al género, 54 \% correspondió al género femenino mientras que $46 \%$ fue para el masculino, en lo concerniente al grado de escolaridad 43.8 
Tabla 1. Nivel de Autoestima en estudiantes de secundaria

\begin{tabular}{|l|c|c|}
\hline \multicolumn{1}{c|}{ Nivel de autoestima } & $\boldsymbol{f}$ & \\
\hline Autoestima Baja & 51 & \\
Autoestima Media & 71 & 15.7 \\
Autoestima Alta & 202 & 62.4 \\
Total & 324 & 100 \\
\hline
\end{tabular}

Fuente: Escala Autoestima de Rosenberg

$\boldsymbol{n}=324$

Tabla 2. Prevalencias de consumo de tabaco en estudiantes de secundaria.

\begin{tabular}{|l|c|c|c|c|c|c|}
\hline \multirow{2}{*}{} & \multicolumn{2}{|c|}{ Si } & \multicolumn{2}{c|}{ No } & \multicolumn{2}{c|}{ IC } \\
\cline { 2 - 7 } & $\boldsymbol{f}$ & $\%$ & $\boldsymbol{f}$ & $\%$ & LI & LS \\
\hline Alguna vez en la vida & 31 & 9.6 & 293 & 90.4 & 6 & 13 \\
En el último año & 9 & 2.8 & 315 & 97.2 & 1 & 5 \\
En el último mes & 6 & 1.9 & 318 & 98.1 & 0 & 3 \\
En los últimos 7 días & 2 & 0.6 & 322 & 99.4 & 0 & 1 \\
\hline
\end{tabular}

Fuente: CDPCT

$\boldsymbol{n}=324$

Tabla 3. Relación entre Niveles de autoestima y consumo de tabaco en estudiantes de secundaria.

\begin{tabular}{|l|c|c|c|}
\hline & Valor & gl & $\boldsymbol{p}$ \\
\hline Chi-cuadrado de Pearson & 1.069 & 2 & 0.59 \\
\hline
\end{tabular}

Fuente: Escala Autoestima de Rosenberg y CDPCT

$\boldsymbol{n}=324$

\% de los participantes cursaban el tercer grado, $34.9 \%$ el segundo grado y $21.3 \%$ refirieron cursar el primer grado en el momento de la entrevista. El 99.4 \% mencionaron dedicarse a estudiar solamente y un dato importante de resaltar es el hecho de que la totalidad de los participantes refirieron vivir con sus padres.

En la Tabla 1 es posible observar que, de acuerdo a los niveles de autoestima clasificados en la escala de Rosenberg, los puntajes obtenidos por el total de los participantes del estudio, ubican al mayor porcentaje de ellos, (62.4 \%) en el rango de autoestima alta, sin embargo, $15.7 \%$ (51) se encontró con nivel de autoestima baja. Adicionalmente, en relación al nivel de autoestima por género, el mayor porcentaje de los adolescentes, tanto del género masculino $(70.5 \%)$ como del femenino (55.4\%) poseen un nivel de autoestima alta, mientras que el nivel de autoestima baja para el género masculino fue $10.1 \%$ (15) y el femenino $20.6 \%$ (36).

En lo que respecta al consumo de tabaco, la Tabla 2 muestra que se encontró un porcentaje muy reducido en la prevalencia de consumo de tabaco alguna vez en la vida, ya que solo el 9.6 \% (31) de los encuestados afirmó haber consumido tabaco en algún momento de su vida, el restante $90.4 \%$ señalo nunca haber consumido tabaco. De igual forma, se observa un importante decremento en el porcentaje de consumo de tabaco en el último mes de $1.9 \%$ (6). Se encontró que la edad de inicio de consumo presentó una media de 12 años ( $D E=1.5)$.

Para responder al tercer objetivo del estudio que establece, comprobar la relación entre la autoestima y el consumo de tabaco. En la Tabla 3 se muestra que no se encontró relación estadísticamente significativa entre los niveles de autoestima y el consumo de tabaco $(p>0.05)$.

\section{DISCUSIÓN}

En el presente estudio fue posible determinar la relación de la autoestima y el consumo de tabaco en una muestra de 324 adolescentes, quienes cursaban sus estudios de nivel secundaria en una institución pública del área urbana, de la cd. de H. Matamoros Tamaulipas, México.

Los hallazgos muestran que la edad de inicio de consumo reportada por los participantes del estudio es a los 12 años, 
dato que es menor a lo señalado por la Encuesta Nacional de Adicciones (2011) ${ }^{(23)}$ al mencionar que el adolescente inicia su consumo en promedio a los 14 años, esta misma encuesta identifica a este grupo poblacional con mayor vulnerabilidad y riesgo ante la epidemia del tabaquismo, ya que el $12.3 \%$ (1.7 millones) de los adolescentes de México son fumadores activos. La literatura describe que el inicio de la educación secundaria marca el principio del consumo experimental de sustancias (24), ya que es en la etapa temprana de la adolescencia cuando el joven empieza a experimentar curiosidad por nuevas sensaciones (25), así como a desafiar y romper las normas sociales establecidas (26).

En relación con el primer objetivo del estudio, el cual señala determinar los niveles de Autoestima en adolescentes de secundaria, en el presente estudio se evidenció que el mayor porcentaje de los adolescentes (62.3\%) se ubicó en el rango de autoestima alta, similar a lo encontrado en un estudio realizado en adolescentes de población mexicana ${ }^{(27)}$. Lo anterior pudiera explicarse en virtud de que la adolescencia es considerada una etapa especialmente relevante para el desarrollo y consolidación de la autoestima. En cuanto a los niveles de autoestima por género, aun cuando el mayor porcentaje de los adolescentes de ambos géneros mostraron un nivel de autoestima alto; en el caso de los masculinos el porcentaje fue mayor (70.5\%), en comparación con las participantes del género femenino (55.4\%). Dato que es congruente con la literatura al señalar que en la infancia los niveles de autoestima son similares en ambos sexos, sin embargo, en la adolescencia emerge un "vacío" entre ellos, presentando los varones una autoestima más elevada y estable en comparación con las mujeres (28). Adicionalmente, investigaciones previas mencionan que la autoestima de los varones adolescentes depende de sus logros tanto personales como sociales, y un alto nivel de autoestima en los adolescentes, se traduce en un efecto positivo de satisfacción vital ${ }^{(29)}$.

Referente al segundo objetivo que enuncia, identificar la prevalencia de consumo de tabaco global, lápsica, actual e instantánea en adolescentes de secundaria, los resultados obtenidos muestran que un porcentaje muy reducido de los encuestados (9.6\%) mencionó haber consumido tabaco alguna vez en la vida; porcentaje que es menor a lo reportado por la Encuesta nacional de adicciones en el $20111^{(23)}$ y por la OMS en el $2015^{(30)}$ quienes señalan que el $12.3 \%$ y $22.0 \%$ respectivamente de los adolescentes son fumadores habituales de tabaco. Sin embargo, es similar a lo reportado por investigadores españoles, quienes estudiaron la impulsividad y el consumo de tabaco en una muestra de adolescentes que cursaban su instrucción secundaria ${ }^{(25)}$. Un dato importante de resaltar es que los resultados del presente estudio muestran que el porcentaje de los adolescentes que reportaron haber consumido tabaco alguna vez en la vida es muy reducido, además de que se observa que el consumo va disminuyendo en cada una de las prevalencias, lo que significa que del total de adolescentes que tienen un primer contacto con el tabaco, no todos se enganchan con un consumo habitual. Lo anterior pudiera deberse a que la edad promedio de los participantes del estudio, es un año menor a la edad de inicio de consumo de tabaco diario reportado por la Encuesta Mundial sobre Tabaquismo en Jóvenes ${ }^{(4)}$, o bien, al hecho de que el total de los adolescentes reportaron vivir con sus padres, lo que condiciona que estén bajo su supervisión, situación que actúa como un factor protector para el consumo de tabaco (24). Otra explicación pudiera ser que, quizá los adolescentes eviten el consumo por percibir al tabaco como algo peligroso para la salud y el rendimiento físico ${ }^{(31)}$.

En cuanto al tercer objetivo que plantea, comprobar la relación entre la autoestima y el consumo de tabaco en adolescentes de secundaria, los resultados documentaron que no se encontró relación significativa entre los niveles de autoestima y el consumo de tabaco. El resultado quizá obedezca a que más del $80.0 \%$ de los participantes reportaron niveles de autoestima alta y media, lo cual la literatura identifica como un factor protector ante el consumo de sustancias ${ }^{(15)}$. Aunado a lo anterior, el total de los adolescentes que participaron en el estudio cohabitaban con ambos padres, lo que de acuerdo a la literatura existente, el mantener una comunicación efectiva con los padres y el apoyo familiar percibido, actúan como un factor protector ante el consumo de tabaco ${ }^{(32,33)}$. De igual forma, se ha identificado una función mediadora de la autoestima y las características del funcionamiento familiar, actuando ambas condiciones como un factor protector ante el consumo de sustancias en los adolescentes. Es decir, un nivel alto de autoestima y un clima familiar positivo disminuye el riesgo de consumo de sustancias en esta población ${ }^{(34)}$.

\section{CONCLUSIONES}

De los resultados obtenidos y en relación con los objetivos del estudio, se puede concluir que: respecto al consumo de tabaco, la prevalencia y la frecuencia en el consumo de tabaco encontrada fue muy baja. Se encontró que el mayor porcentaje de los participantes en el estudio, mostraron niveles de autoestima alto. Referente a los niveles de autoestima por género, el mayor porcentaje de los adolescentes de ambos géneros mostraron un nivel de autoestima alto, destacando el género masculino, y en cuanto al grado escolar; los porcentajes más elevados se identifican en los alumnos del tercer grado. No se encontró correlación significativa de la autoestima y el consumo de tabaco alguna vez en la vida.

\section{RECOMENDACIONES}

Aun cuando los resultados del presente estudio evidenciaron un porcentaje muy reducido en el consumo de tabaco, se considera necesario continuar e incrementar el diseño 
de intervenciones que promuevan y refuercen conductas saludables en los adolescentes. Implementar estrategias que permitan al adolescente analizar y concientizarse sobre las consecuencias negativas del consumo de tabaco a temprana edad, así como desarrollar acciones permanentes de prevención y promoción de la salud en esta población consideraba como vulnerable por las características propias de la etapa de la adolescencia.

\section{CONFLICTO DE INTERESES}

Los autores declaran no tener conflicto de intereses.

\section{FINANCIAMIENTO}

Nuestro agradecimiento al Programa para el Desarrollo Profesional Docente para el Tipo Superior (PRODEP/25530) por el financiamiento otorgado para el desarrollo de la presente investigación.

\section{REFERENCIAS BIBLIOGRÁFICAS}

1. Nuño-Gutiérrez BL, Celis-de la Rosa A, García-Pérez OP. Indicadores de salud mental y su asociación con tabaquismo en adolescentes de Guadalajara según sexo. Rev Méd Inst Mex Seguro Soc [Internet]. 2015 [consultado Septiembre 2019]; 53(5): 570-577. Disponible en: https:// www.medigraphic.com/cgi-bin/new/resumen.cgi?IDARTI$\mathrm{CULO}=62819$

2. Organización Mundial de la Salud (OMS). Tabaco [Internet]. OMS. 2018 [consultado Junio 2019]. Disponible en: https://www.who.int/es/news-room/fact-sheets/detail/ tobacco

3. Nagrath D, Mathur R, Gupta R, Zodpey SM. Tobacco use in adolescence: Influence of socio-demographic and socioeconomic factors. Preventive Medicine Reports [Internet]. 2019 [consultado Mayo 2019]; 14:1-4. Disponible en: https://doi.org/10.1016/j.pmedr.2019.100832

4. Organización Panamericana de la Salud (OPS). Encuesta Mundial de Tabaquismo en Jóvenes (EMTJ) [Internet]. OPS. 2016 [consultado Junio 2019]. Disponible en: https://www. paho.org/hq/index.php?option=com_content\&view $=$ article\&id=1748:2009-global-youth-tobacco-survey-gyts\&ltemid=1185\&lang $=$ es

5. Cogollo-Milanés Z, Gómez-Bustamante EM. Variables asociadas al inicio del consumo de cigarrillo en adolescentes estudiantes de básica secundaria de los colegios oficiales de la ciudad de Cartagena, Colombia. Aquichan
[Internet]. 2014 [consultado Agosto 2019]; 14(2): 226-23. Disponible en: https://www.redalyc.org/pdf/741/74131358009.pdf

6. Comisión Nacional Contra las Adicciones. Encuesta Nacional de Consumo de Drogas, Alcohol y Tabaco (ENCODAT) 2016-2017 [Internet] ENCODAT 2016. [ consultado Mayo 2019]. Disponible en: https://www.gob.mx/salud\%7Cconadic/acciones-y-programas/encuesta-nacional-de-consumo-de-drogas-alcohol-y-tabaco-encodat-2016-2017-136758

7. Martínez-Hernáez A, Marí-Klose M, Juliá A, Escapa S, Marí-Klose P, DiGiacomo S. Consumo diario de tabaco en la adolescencia, estados de ánimo negativos y rol de la comunicación familiar. Gac Sanit [Internet]. 2012 [consultado Octubre 2019]; 26(5): 421-428.

Disponible en: https://doi.org/10.1016/j.gaceta.2011.09.030

8. Schoeps K, Tamarit A, González R, Montoya-Castilla I. Competencias emocionales y autoestima en la adolescencia: impacto sobre el ajuste psicológico. Revista de Psicología Clínica con Niños y Adolescentes [Internet]. 2019 [consultado Mayo 2020]; 6(1): 51.56. Disponible en: https://doi: 10.21134/rpcna.2019.06.1.7

9. Veeranki SP, Mamudu HM, John RM. Ouma, AEO. Prevalence and correlates of tobacco use among school-going adolescents in Madagascar. Journal of Epidemiology and Global Health [Internet]. 2015 [consultado Julio 2019]; 5: 223-247. Disponible en: https://dx.doi.org/10.1016/j.jegh.2014.12.005

10. Ariza C, García-Continente X, Villalbí JR, Sánchez-Martínez F, Pérez A, Nebot M. Consumo de tabaco de los adolescentes en Barcelona y tendencias a lo largo de 20 años. Gac Sanit [Internet]. 2014 [consultado Agosto 2019]; 28(1): 24-33. Disponible en: http://dx.doi.org/10.1016/j.gaceta.2013.08.005

11. Villatoro VJA, Mendoza MMA, Moreno LM, Oliva RN, Fregoso ID, Bustos GM, Medina-Mora IME. Tendencias del uso de drogas en la Ciudad de México: Encuesta de estudiantes, octubre 2012. Salud Ment [Internet]. 2014 [consultado junio 2019]; 37: 423-435. Disponible en: http://www.scielo.org.mx/scielo. php?script=sci_arttext $\&$ pid $=$ S0185-33252014000500009

12. González-González A, Juárez GF, Solís TC, González-Forteza, Jiménez TA, Medina-Mora ME, Fernández-Varela, $\mathrm{MH}$. Depresión y consumo de alcohol y tabaco en estudiantes de bachillerato y licenciatura. Salud Ment [Internet]. 2012 [consultado Mayo 2019]; 35(1): 51-55. Disponible en: http://www.scielo.org.mx/scielo.php?script=sci_arttext\&pi$d=S 0185-33252012000100008$

13. Álvaro GJl, Zurita OF, Castro SM, Martínez MA, García SS. Relación entre consumo de tabaco y alcohol y el autoconcepto en adolescentes españoles. Rev Complut Educ [Internet]. 2016 [consultado Julio 2019]; 27(2): 533-550. Disponible en: 
http://dx.doi.org/10.5209/rev_RCED.2016.v27.n2.46605

14. Rosenberg, M. Society and the adolescent self-imagen. Princeton, NJ: Princeton University Press. 1965. 326.

15. Liberini S, Rodríguez GE. Romero NA. El rol de la autoestima, la personalidad y la familia en el consumo de alcohol en la adolescencia. PSOCIAL [Internet]. 2016 [consultado junio 2019]; 2(1): 27-37. Disponible en: https://publicaciones.sociales.uba.ar/index.php/psicologiasocial/article/view/1469

16. Anbarlouei M, Sarbakhsh P, Dadashzadeh H, Ghiasi A, Ataeias M, Dorosti A. Mohammadpooras A. Cigarette and hookah smoking and their relationship with self-esteem and communication skills among high school students. Health Promot Perspect [Internet]. 2018 [consultado Agosto 2019]; 8(3): 230-236. Disponible en: doi: 10.15171/hpp.2018.31

17. Ortiz LMC, Gogeascoechea-Trejo MC, Blázquez-Morales MSL, Pavón-León P, Barreto-Bedoya P, Pérez-Prada, M. P. Factores psicosociales asociados al consumo de drogas en estudiantes de dos universidades de América Latina. Revista Salud Pública [Internet]. 2018 [consultado Agosto 2019]; 22(1): 41-52. Disponible en: https://doi.org/10.31052/1853.1180.v22. n1.18655

18. Isorna FM, Golpe S, Otero RM, Ayesta AJ, Gómez SP. Consumo de tabaco y autoestima en adolescentes con y sin Trastorno por Déficit de Atención con Hiperactividad (TDAH): propuestas para una mejor prevención. Revista española de Drogodependencias [Internet]. 2018 [consultado Julio 2019]; 43(4): 69-82. Disponible en: https://doctorsergiocalvo.com.ar/ wp-content/uploads/43921.pdf

19. Riquelme M, García OF, Serra E. Desajuste psicosocial en la adolescencia: socialización parental, autoestima y uso de sustancias. Anales de Psicología [Internet]. 2018 [consultado Agosto 2019]; 34(3): 536-544. Disponible en: doi: https://doi. org/10.6018/analesps.34.3.315201

20. Telumbre-Terrero JY, López-Cisneros MA, Noh-Moo PM, Villanueva JR, Torres-Obregón R. Autoestima y consumo de alcohol en adolescentes de secundaria de Ciudad del Carmen, Campeche. Sanus [Internet]. 2018 [consultado Mayo 2020]; 3(6): 20-31.

21. Jurado CD, Jurado CM, López VK, Querevalú GB. Validez de la Escala de Autoestima de Rosenberg en universitarios de la Ciudad de México. Revista Latinoamericana de Medicina Conductual [Internet]. 2015 [consultado Mayo 2020]; 5(1): 1822.

22. Secretaría de Salubridad y Asistencia (SSA). Reglamento de la Ley General de Salud en Materia de Investigación para la Salud [Internet]. SSA. 1987 [consultado Mayo 2019]. Disponible en: http://www.salud.gob.mx/unidades/cdi/nom/compi/ rlgsmis.html

23. Encuesta Nacional de Adicciones (ENA) Encuesta Nacional 2011 de Adicciones Tabaco [Internet]. ENA. 2011. Disponible en: https://www.insp.mx/images/stories/Produccion/pdf/130911_ENAtabaco.pdf

24. Pérez-Milena A, Martínez-Fernández ML, Redondo-Olmedilla M, Álvarez NC, Jiménez PI, Mesa GI. Motivaciones para el consumo de tabaco entre los adolescentes de un instituto urbano. Gac Sanit [Internet]. 2012 [consultado Mayo 2019]; 26(1): 51-57. Disponible en: doi:10.1016/j. gaceta.2011.03.021

25. Pérez-Fuentes MC, Gázquez JJ, Molero MM., Cardila F, Martos Á, Barragán AB, Mercader I. Impulsividad y consumo de alcohol y tabaco en adolescentes. Eur. J. Investig. Health Psychol. Educ [Internet]. 2015 [consultado Julio 2019]; 5(3): 371-382. Disponible en: doi: 10.1989/ejihpe. v5i3.139

26. Rodríguez PLA, Alonso CBA, Alonso CMM, Alonso CMT, Armendáriz GNA, Oliva R. NN. Consumo de alcohol y tabaco en adolescentes. Revista ESMAD [Internet]. 2016 [consultado Junio 2019]; 12(4), 200-206. Disponible en: doi:10.11606/issn.1806-6976.v12i4p200-206

27. Gámez-Medina ME, Guzmán-Facundo, FR, Ahumada-Cortez JG, Alonso-Castillo MM, Gherardi-Donato DEC. Autoestima y consumo de alcohol en adolescentes escolarizados. Nure Inv [Internet]. 2017 [consultado Mayo 2019]; 14(88): 1-9. Disponible en: https://dialnet.unirioja.es/ servlet/articulo?codigo $=6278118$

28. Rodríguez NC, Caño GA. Autoestima en la adolescencia: análisis y estrategias de intervención. IJP\&PT [Internet]. 2012; 12(3): 389-403. Disponible en: http://www.redalyc. org/articulo.oa?id=56024657005

29. San-Martin JL, Barra E. Autoestima, Apoyo Social y Satisfacción Vital en Adolescentes. Ter Psicol [Internet]. 2013 [consultado Julio 2019]; 31(3): 287-291. Disponible en: https://scielo.conicyt.cl/scielo.php?pi$d=$ S0718-48082013000300003\&script=sci_arttext\&tln$\mathrm{g}=\mathrm{en}$

30. Organización Mundial de la Salud (OMS). Informe sobre la epidemia mundial del tabaquismo [Internet]. OMS. 2015. [consultado Mayo 2019]. Disponible en: https://www. who.int/tobacco/global_report/2015/summary/es/

31. De los Reyes NLR, Moreno RVM, Vásquez SMG, Vázquez TMGE, Ramírez HSA, Ramos LSJ. Comportamiento del adolescente ante el consumo de tabaco. RICS [Internet]. 2016 [consultado Octubre 2019]; 5(10): np. Disponi- 
ble en: https://dialnet.unirioja.es/servlet/articulo?codigo $=5644371$

32. Cheesman MSS, Suárez LN. Factores de riesgo y protectores del tabaquismo en estudiantes de la Facultad Ciencias Médicas USAC, Guatemala. Horizonte Sanitario [Internet]. 2014 [consultado Julio 2019]; (13)3: 223-232. Disponible en: https://www.redalyc.org/pdf/4578/457845147002.pdf

33. Molero-Jurado MM, Pérez-Fuentes M, Gázquez-Linares JJ, Barragán-Martín AB. Análisis y perfiles del consumo de drogas en adolescentes: percepción del apoyo familiar y valoración de consecuencias. Atención Familiar [Internet]. 2017 [consultado Agosto 2019]; 24(2): 56-61. Recuperado de: https://doi.org/10.1016/j.af.2017.02.001

34. Jiménez TI. Autoestima de Riesgo y Protección: Una Mediación entre el Clima Familiar y el Consumo de Sustancias en Adolescentes. Psychosocial Intervention [Internet]. 2011 [consultado Julio 2019]; 20(1): 53-61. Recuperado de: doi: 10.5093/in2011v20n1a5 